\title{
Claves para la gestión de puestos de trabajo en las organizaciones en red
}

\author{
Key issues to manage work positions in networked organizations
}

\author{
Ana Moreno Romero ', Carlos Mataix Aldeanueva ' y Ángel Mahou Fernández² \\ I Escuela Técnica Superior de Ingenieros Industriales. \\ Universidad Politécnica de Madrid. C/José Gutiérrez Abascal, 2,28006 Madrid. \\ 2 Departamento de Recursos Humanos de Red Eléctrica de España. \\ ana.moreno.romero@upm carlos.mataix@upm.es
}

Fecha de recepción: 16-04-2010

Fecha de aceptación: 29-10-2010

Resumen: Las organizaciones viven cambios estratégicos, culturales y organizativos para adaptarse a la sociedad red. La incorporación de las Tecnologías de Información y Comunicación facilita los esquemas de trabajo en red dentro de las organizaciones y entre ellas. En este contexto, hay profesionales desbordados en su puesto de trabajo, por exceso de información, correos electrónicos, llamadas, reuniones, plataformas virtuales.Estos cambios suponen un reto para la organización del trabajo y mejora de productividad. En este artículo se propone un marco de análisis para esta organización del trabajo en puestos caracterizados por el predominio del conocimiento. Finalmente se contrasta con un estudio de caso.

Palabras clave: organización en red, redes, organización virtual, trabajador en red, puesto de trabajo.

Abstract: Organizations face strategic, cultural and organizational changes to evolve towards information society. Information and Communication Technologies boost networked jobs inside organizations and among them. In this context, professionals are overwhelm with big amount of information, mails, phone calls, meetings or virtual platforms, what represents a new challenge for work organization and productivity. This paper makes a proposal of a framework for analysing work organization in knowledge based positions, and includes a case study to evaluate it.

Keywords: networked organizations, network, virtual organization, teleworker, work position.

\section{Las organizaciones en la sociedad en red}

Las organizaciones viven cambios estratégicos, culturales y organizativos para adaptarse a la sociedad red. La incorporación de las Tecnologías de la Información y la Comunicación (TIC) facilita trabajo en red de los profesionales (teletrabajo, participación en equipos virtuales, movilidad....) y la relación entre organizaciones (redes inter-organizativas, alianzas virtuales, proyectos compartidos...).

El objeto de este artículo es ofrecer un marco conceptual para las redes organizativas, a partir del cual sea posible proponer un modelo de análisis aplica- ble a la organización de los puestos de trabajo basados en conocimiento. Para entender los procesos de cambio que afectan a las organizaciones en red se han seleccionado cinco pilares teóricos que explican las dificultades a las que se enfrentan los trabajadores inmersos en modelos en red:

- Ámbitos de transformación a redes (fig. I).

- Gestión del conocimiento como estructura de los procesos organizativos,

- El modelo de planos de avance intra-organizativo hacia modelos red (fig. 2). 
- Impacto en los esquemas de trabajo en los grupos (por ejemplo la incorporación del teletrabajo).

- El impacto en las competencias de los trabajadores y trabajadoras.

En los siguientes epígrafes se desarrollan brevemente estos conceptos para sustentar la propuesta de modelo de análisis para los puestos de trabajo del conocimiento.

\section{Redes de organizaciones}

El diccionario de la RAE define red como un «conjunto sistemático de nexos o de hilos conductores o de vías de comunicación o de agencias y servicios para determinado fin». Al hablar de redes entre organizaciones, estos nexos y vías de comunicación sirven para vincular a varias organizaciones y se ponen al servicio de sus misiones, estrategias y objetivos. Desde esta perspectiva tan amplia, la noción de red puede encontrarse aplicada en entornos muy variados: Internet como red de redes, redes sociales, capital social en red, redes de pymes, redes ciudadanas, I+D tecnológico en red...

Figura I

Modelo de ámbitos de transformación a redes

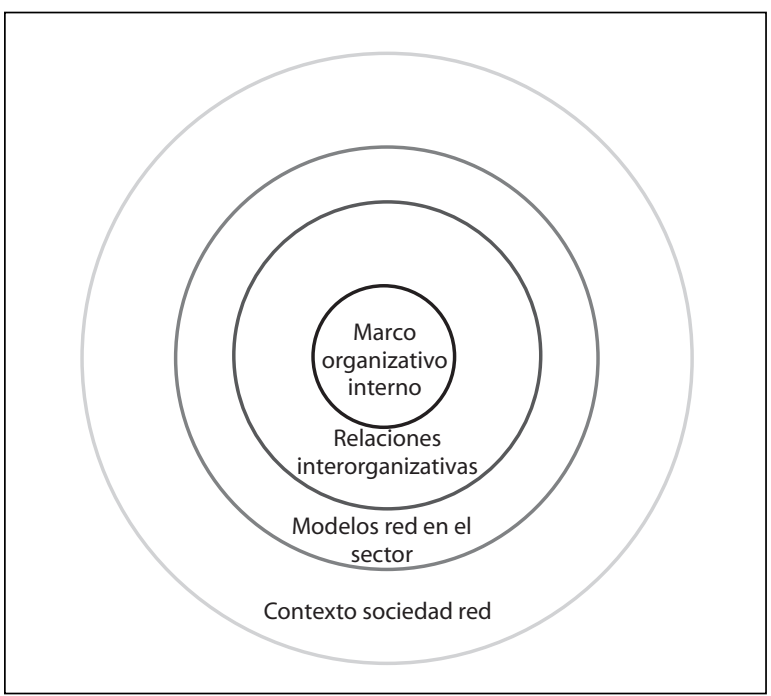

El avance a modelos de funcionamiento en red se produce en cuatro ámbitos que evolucionan simul- táneamente y no siempre alineados: la propia organización, el conjunto de organizaciones con el que se interactúa, el sector de actividad y el conjunto de la sociedad (Moreno, 2009 '). Podría afirmarse que, en el entorno actual, prácticamente cualquier organización trabaja en red desde algún tramo de su cadena de valor, si bien la profundidad de la participación es muy variable; por ejemplo, una multinacional de telecomunicaciones trabaja en un entorno red mucho más avanzado que un pequeño comercio en una zona rural de España.

\section{Los procesos en las organizaciones y la gestión del conocimiento}

Desde la publicación del «Principios de la administración científica», de Taylor ( $|9| \mid$ ), pasando por las teorías de McGregor o de Tavistoke que amplían las actividades de un sistema a los procesos psicológicos, sociales y tecnológicos, hasta los actuales cambios hacia modelos en red, la organización del trabajo ha sido una disciplina que ha intentado aportar un examen sistemático de los métodos empleados para realizar actividades, con el fin de mejorar la utilización eficaz de los recursos, y de establecer normas de rendimiento con respecto a las actividades que se están realizando.

Las etapas tradicionales del estudio del trabajo (selección de trabajos relevantes, registro y examen de actividades, creación y evaluación de mejoras, definición e implantación de los procesos asociados, y control), desarrollados en las organizaciones industriales de la segunda mitad del siglo XX, son cada vez más restringidas a operaciones de transformación material (cadenas de montaje) y difíciles de identificar, acotar, observar y estandarizar en las organizaciones del conocimiento.

La nueva columna vertebral para el establecimiento de procesos es la gestión del conocimiento. Los sistemas de información y de gestión del conocimiento buscan, en última instancia, aportar valor a las organizaciones. Si el conocimiento fluye en el día a día de éstas, se puede hablar de la organización que aprende. Luthans (2008) habla de tres factores para que este alineamiento organizativo en torno al conocimiento se produzca:

\footnotetext{
I Un resumen del planteamiento de esta línea de investigación ha sido publicado en la revista Telos ( $n^{\circ} 81$, noviembre 2009), a la que queremos agradecer la oportunidad de participar en la edición especial de 25 aniversario.
} 
- Presencia de tensión: brecha entre la visión y la realidad, cuestionamiento/investigación, desafío al statu quo, reflexión crítica.

- Pensamiento de sistemas: visión compartida, pensamiento holístico, apertura.

- Cultura que facilita el aprendizaje: sugerencias, trabajo en equipo, delegación de autoridad y empatía.

¿Cómo se analizan estos procesos organizativos para mejorar la productividad? ¿Se consigue mayor eficiencia en entornos organizativos en red? No es fácil contestar estas preguntas porque las organizaciones están en medio de un proceso de transformación en el que conviven esquemas de trabajo tradicionales con avances a modelos en red.

De manera simplificada podría decirse que las organizaciones están avanzando en paralelo en tres planos: la incorporación de las TIC, la transformación de los modelos internos de trabajo y la participación, como organización, en redes más amplias (Figura 2).

Figura 2

Modelo de planos de avance intra-organizativo hacia modelos red

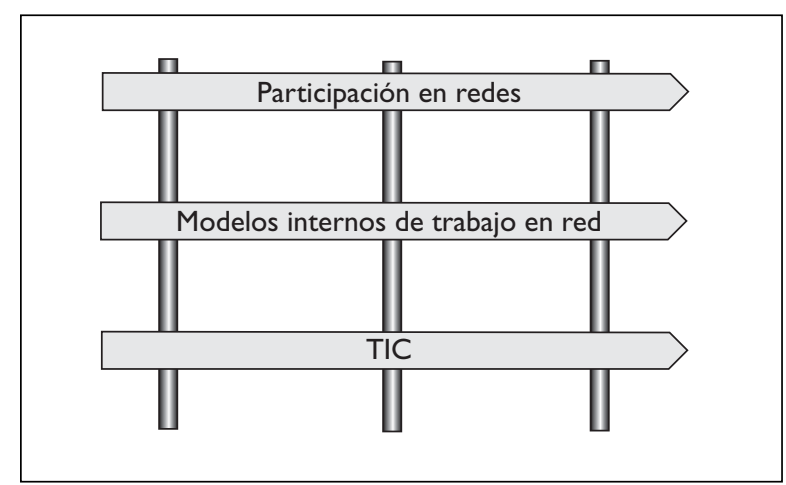

Las empresas, las Administraciones Públicas, las ONG, las universidades tienen distintos niveles de avance en los tres planos, pero en muchos casos se están dando los primeros pasos y existen desajustes entre los niveles.

\section{Nuevos esquemas de cohesión en los grupos de trabajo}

Las organizaciones de la era industrial se caracterizan por tener centros de trabajo definidos, en los que se comparte un espacio, un horario y una cultura. El compromiso con la organización se alcanza con pro- cesos de socialización adaptados a este marco. Los pilares de cohesión organizativa: cultura, tiempo y espacio compartido, podrían debilitarse en los modelos de trabajo en red, tanto entre organizaciones, como dentro de la organización. En este nuevo contexto, el sistema de significados cambia, y la identidad social de los trabajadores se ve afectada (Wiesenfeld, Raghuram, y Garud, 1998).

El teletrabajo ha sido la actividad que ha aglutinado las primeras experiencias de deslocalización espacial y temporal, y tiene ya una historia de 30 años de experiencias evaluables, especialmente entre las grandes empresas. Las empresas del sector TIC son pioneras en la implantación de teletrabajo y por eso también lo son en el desarrollo de modelos para analizar el impacto en la organización y en los profesionales.

La extrapolación a otro tipo de organización como la Administración Pública, Pyme u ONG, no es automática, aunque todas las organizaciones evolucionan a un cierto nivel de trabajo en red vinculado a la movilidad y flexibilidad que las TIC permiten.

Para entender el impacto en las organizaciones de la incorporación de las TIC y los nuevos modelos de trabajo en red, hay que analizar el comportamiento de los grupos humanos que las integran. Es en este nivel donde puede observarse el que pudiera ser el cambio más relevante vinculado a la penetración de las TIC: la modificación en los esquemas de comunicación y, directamente relacionado, la construcción de identidad en el grupo. En este sentido se ha observado como el cambio en los canales de comunicación puede tener efectos profundos en la forma de funcionamiento de los grupos (Hollingshead, 200 I). Esta autora analiza la comunicación electrónica e identifica los siguientes factores clave:

- La importancia de la comunicación verbal.

- El impacto del conocimiento previo entre las personas.

- La transparencia en la compartición de información.

- La permanencia del estatus en comunicaciones virtuales.

- El nivel de adaptación de las personas a los nuevos canales.

Las TIC que transforman la comunicación están en un proceso continuo de innovación y, por tanto, la 
adaptación de los usuarios ha de ser también continua. Hasta que no transcurre un cierto tiempo no se sabe si se ha producido un cambio real en las formas de trabajo y relación, o se trata de una simple adaptación a un nuevo instrumento. De lo que no hay duda es de que, en los grupos de trabajo, la comunicación electrónica tiene un papel central que todavía no ha sido suficientemente estudiado.

\section{Las competencias de los profesionales en el trabajo en red}

Los profesionales incorporan las tecnologías en sus vidas y necesitan saber qué son los sistemas de información, cuáles son las nuevas formas de trabajar y como manejar grandes cantidades de información para utilizar adecuadamente las muchas facilidades de comunicación y organizar de una forma diferente el tiempo. Las potenciales ventajas que traen las TIC, se van concretando en experiencias de éxito contrastado: el teletrabajo, las redes sociales profesionales, el acceso a la información que se necesite de manera inmediata, la comunicación con las personas del entorno profesional y personal por diferentes canales (skype, correo electrónico, sms, videoconferencia, Messenger, voz en movilidad. ..) o la participación en grupos virtuales. Los equipos de trabajo en movilidad son cada vez más habituales y el teléfono móvil el terminal de acceso a una parte creciente de los servicios de red. La experiencia organizativa acumulada en la incorporación del móvil muestra que hay un proceso de aprendizaje en la interiorización de la tecnología (Iglesias-Pradas et als, 2009).

Sin embargo, hay que prestar atención a algunos riesgos no menores:

- El espejismo de la multiplicación del tiempo que nace de la mayor flexibilidad para su gestión.

- El exceso de información.

- La fatiga de red por el crecimiento del número de contactos activos.

- La ficción de que en grupos virtuales se consiguen iguales resultados, con iguales métodos, que en grupos «cara a cara».

Disfrutar de las oportunidades y afrontar adecuadamente los desafíos requiere un nuevo ámbito de competencias. Podría incluso decirse que las claves para la adecuada «alfabetización digital», se basan en las transformación de los procesos sociales de aprendizaje (Casado, 2006); Santos et als., 2009.

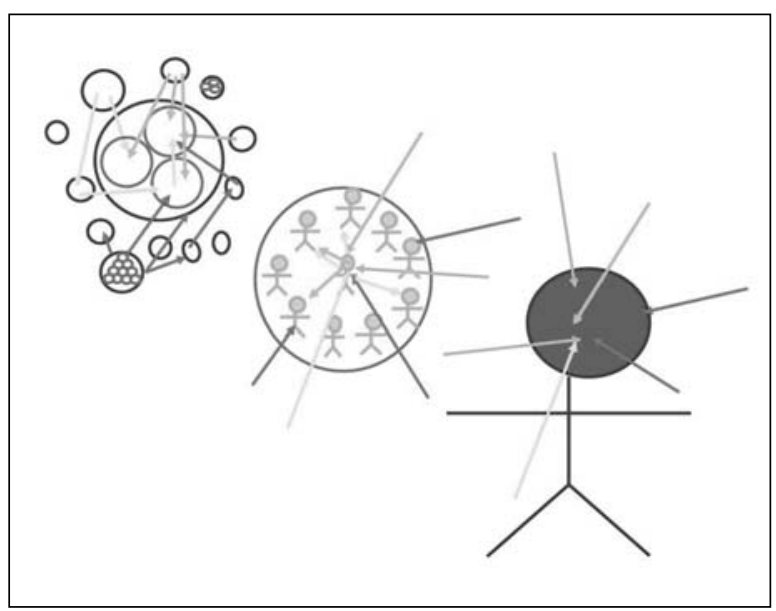

La información que reciben los profesionales para el desarrollo de sus funciones y su crecimiento profesional en el entorno tradicional, con una estructura piramidal tanto dentro de la organización como en la relación entre organizaciones, se rige por esquemas claros y ordenados. Pero si se analiza un escenario más complejo, en el que todos los niveles operan con modelos en red, la cantidad de inputs que tiene, recibe, interpreta y utiliza un profesional se multiplica y los instrumentos para alinear las actuaciones dentro de una organización ya no pueden ser las instrucciones individuales, sino la compartición de unos objetivos y de unos valores que permitan una razonable convergencia en procesos y en decisiones que son paralelos y autónomos.

Si se analiza cómo facilitar el funcionamiento en red, se pueden enunciar nuevos mapas de competencias que los profesionales necesitan (Moreno, 2009):

- Las habilidades red, para utilizar adecuadamente el nuevo entorno tecnológico: gestión de la información, administración de correo electrónico, comunicación electrónica, coordinación de equipos en red, el arte del tiempo asíncrono...

- Las llamadas capacidades generativas para aportar valor en el entorno de redes organizativas y sociales: competencias individuales y sociales, competencias para el trabajo en grupo y pensamiento sistémico para el contexto en red.

- Equilibrio de roles, para la gestión armónica de este nuevo contexto complejo y carente de pautas fijas: centramiento dinámico, simplificación de la operación y control de la atención. 
El liderazgo adaptado a la administración de estas competencias en los equipos, es uno de los desafíos principales en las organizaciones que van avanzando a modelos en red. Este perfil de líder tan exigente, requiere de nuevas herramientas organizativas. Las ideas recogidas en este epígrafe confirman que los modelos organizativos y los procesos de trabajo tradicionales no son fácilmente extrapolable a los puestos del conocimiento.

\section{Organización del trabajo en puestos del conocimiento}

La gestión de equipos en organizaciones en red requiere de la integración de todos los ingredientes mencionados, para que el individuo pueda entender cuál es su puesto de trabajo y organizar su día a día con un cierto nivel de claridad y confort.

Sennett (1998) enuncia los puntos críticos que amenazan a los trabajadores de entornos flexibles y que les lleva a lo que el autor denomina «la corrosión del carácter» y la superficialidad de la vida laboral:

- La ilegibilidad del proceso en el que se participa por exceso de fragmentación en la tarea.

- La asunción por parte de los trabajadores de riesgos derivados del cambio y la incertidumbre.

- La volatilidad de los planes y la relativización de éxitos y fracasos con la consiguiente pérdida de sentido y recorrido.

- El excesivo protagonismo del grupo frente a la tarea individual.

«El trabajo en equipo hace hincapié en la capacidad de adaptación del equipo a las circunstancias. Trabajo en equipo es la ética del trabajo que conviene a una economía política flexible ...La antigua ética se fundaba en el uso autodisciplinado del propio tiempo, con el acento puesto en una práctica autoimpuesta y voluntaria más que una sumisión meramente pasiva a los horarios y a la rutina.»

La organización del trabajo para puestos del conocimiento es la clave para el efectivo y satisfactorio trabajo en equipo en modelos en red, y quizás se requiere recuperar parte de esa antigua ética de la que habla Sennett.

En su libro «Diseño de organizaciones eficientes» (1992) Minzberg hablaba de cinco posibles sistemas de coordinación (ajuste mutuo, supervisión directa, estandarización de procesos, estandarización de producciones, estandarización de conocimientos) que usados adecuadamente permiten la consistencia y armonía de las partes de una organización (nivel directivo estratégico, tecno-estructura, staff de apoyo, línea media y núcleo operativo). De las posibles estructuras, (estructura simple, burocracia mecánica, burocracia profesional, forma divisional, adhocracia u organización innovadora, misionera y política) la que parece más adaptada a la organización en red es la adhocrática, pensada para sistemas dinámicos y complejos. La orientación básica es el «intraemprendizaje» porque la innovación se apoya en las iniciativas estratégicas de los profesionales y traslada el poder a los expertos. Para innovar hay que romper los modelos existentes, por lo que esta estructura no puede apoyarse en la estandarización para la coordinación, sino que debe ser capaz de aunar distintos tipos de conocimiento. Además la coordinación se legitima desde el conocimiento experto y no desde la autoridad: «Los directores en las adhocracias raramente gestionan en el sentido habitual de dar instrucciones; en cambio pasan gran parte del tiempo actuando de conectores para coordinar lateralmente el trabajo entre equipos y unidades»...»deben ser maestros en relaciones humanas, capaces de persuadir, negociar, aunar, legitimar y entusiasmar para que los expertos individuales se fundan con armonía en equipos que funcionan» (Minzberg, 89). Uno de los desafíos más complejos para los directivos en este entorno, es garantizar una carga de trabajo estable balanceando los nuevos proyectos, sus esquemas operativos y las asignaciones individuales. No obstante, cualquier estructura organizativa, no sólo la adhocrática, puede incorporar mecanismos de trabajo en red.

Actualmente las organizaciones están estudiando y mejorando los procesos de su cadena de valor, la utilización de las TIC, los sistemas de gestión de conocimiento, las competencias en entornos virtuales, la comunicación con grupos de interés y la productividad de sus trabajadores. Sin embargo, no se avanza en la misma medida en lo que tiene que ver con la definición del puesto de trabajo en relación a la estructura organizativa. Los principios básicos tradicionales como la dirección por objetivos, la cultura de confianza y las políticas de gestión por competencias son difíciles de trasladar a la práctica, y el protagonismo que recae en los directivos y directivas, para su interpretación, es cada vez mayor.

La integración armónica en el puesto de trabajo de los esquemas en red es, sustancialmente, un desafío que afronta cada profesional y para el que, en mu- 
chas ocasiones, no cuenta con pautas claras. Cada vez es más habitual encontrar a personas desbordadas por un exceso de información y demandas de contacto.... imejora la productividad? ¿se va a modelos de hiperproductividad operativa e hipoproductividad estratégica? ¿es sostenible un nivel de fragmentación de la atención como el que viven una proporción creciente de los profesionales? ¿es razonable que cada uno traslade a su puesto de trabajo su estilo personal sin tener en cuenta el impacto en la productividad que conlleva?

Esta compleja y dinámica realidad, está aportando mucha innovación organizativa, pero también está planteando aspectos en la gestión cotidiana de difícil solución. Quizás el mejor símbolo de esta paradoja sea un puesto de trabajo, ocupado por un profesional que está desbordado, trabaja 10 horas al día, no consigue tener nunca sus tareas pendientes resueltas y tiene un alto nivel de estrés. Resulta muy complejo discernir si se trata de un problema de carga de trabajo o de competencia para el desempeño del puesto; si la carga es razonable y tiene las competencias requeridas pero su espíritu creativo o perfeccionista complica las tareas de forma innecesaria; si todo es correcto pero las ineficiencias del grupo o proceso impactan en su carga de trabajo o si el estilo de su director es errático y no da pautas estables.... Los principios teóricos del liderazgo en red son sólidos: el director de equipos debe generar un entono claro y estimulante, ser el mentor de los miembros de su equipo para que salga lo mejor de cada uno, tener una visión estratégica amplia que canaliza de forma ordenada hacia abajo, a la vez que escucha y entiende la información que viene de su equipo y de su entorno. Quizás, este perfil 10, requiera de nuevas herramientas organizativas para actuar en la práctica.

La investigación en torno a la organización del trabajo en puestos del conocimiento puede ayudar a construir un puente entre la integración horizontal de procesos de la cadena de valor (intra e interorganizativa), las herramientas de productividad individual y grupal y, lo que hoy es una carencia, las pautas para la integración individual en los puestos de trabajo.

\section{Construcción de un marco teórico para la organización del trabajo en puestos del conocimiento}

En el grupo de investigación de Organizaciones Sostenibles, del Departamento de Ingeniería de Organización, Administración de Empresas y Estadística de la UPM, se está investigando un modelo de organización del trabajo en puestos del conocimiento. El objetivo de este modelo es ayudar a las organizaciones, a los directores y directoras de quipos y a los trabajadores a organizar mejor puestos de trabajo red. Se basa en tres elementos.

El primero es el modelo CUBOT, que cataloga los puestos de trabajo de acuerdo a tres variables: el nivel de desmaterialización de la actividad (trabaja con átomos o con bits), la flexibilidad que las tareas permiten para la gestión de tiempos y ubicación, y el nivel de complejidad del conocimiento asociado al puesto (modelo CUBOT).

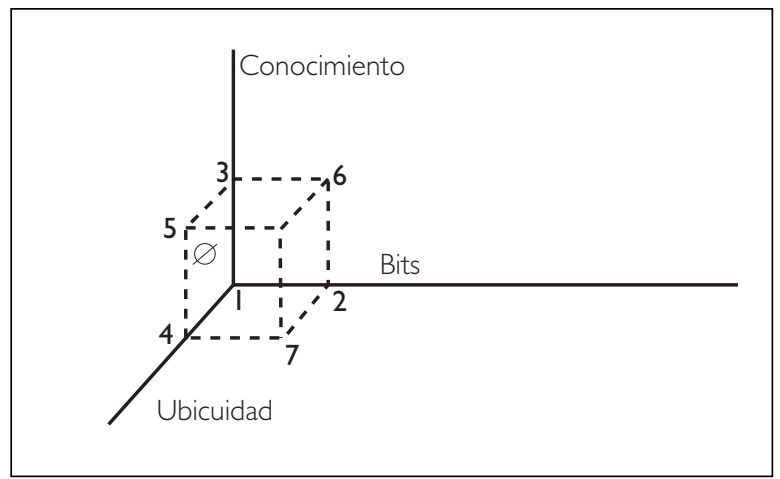

La combinación de estas variables define ocho puestos tipo, con distintos requerimientos funcionales, de integración en los procesos globales, de gestión del conocimiento, claves para el trabajo en equipo y competencias de las personas que ocupan el puesto.

Con esta caracterización, las organizaciones pueden identificar que puestos de trabajo son más cercanos a modelos red.

Los puestos característicos de la sociedad en red son los CUB (conocimiento, ubicuidad y bits), y la caracterización de oportunidades, desafíos y competencias necesarias para trabajadores en red que se ha desarrollado en este artículo, se puede esperar que sea habitual en estos puestos.

El segundo elemento que forma parte del modelo de investigación de organización del trabajo en puestos del conocimiento es una metodología de planificación y control de tiempos, denominada planificación integrada de tiempos (PIT), basada en métodos clásicos de control de proyectos de consultoría. Esta metodología da pautas a los trabajadores para la planificación, contabilización y evaluación del tiempo asignado a las distintas tareas que se atienden en el puesto de trabajo. 


\begin{tabular}{|l|l|}
\hline \multicolumn{1}{|c|}{ Puesto según variables modelo CUBOT } & \multicolumn{1}{c|}{ Caracterización del puesto } \\
\hline I.- CERO & Se trabaja con átomos en esquema fijo con bajo conocimiento \\
\hline 2.- BITS & $\begin{array}{l}\text { Se trabaja con un cierto nivel de desmaterialización (átomos o bits) } \\
\text { de la tarea en esquema fijo con bajo conocimiento de la tarea }\end{array}$ \\
\hline 3.- CONOCIMIENTO & $\begin{array}{l}\text { Se trabaja con cierto nivel de conocimiento de la tarea (seguimiento } \\
\text { o creatividad) en esquema fijo y con átomos }\end{array}$ \\
\hline 4.- UBICUIDAD & $\begin{array}{l}\text { Se trabaja con cierto nivel de flexibilidad en la realización de la ta- } \\
\text { rea(esquema fijo o esquema móvil) con un nivel bajo de conoci- } \\
\text { miento necesario para su desarrollo y con átomos }\end{array}$ \\
\hline 5.- CONOCIMIENTO+ UBICUIDAD & $\begin{array}{l}\text { Se trabajo con átomos en esquema móvil y con alto conocimiento } \\
\text { de la tarea }\end{array}$ \\
\hline 6.- BITS+CONOCIMIENTO & $\begin{array}{l}\text { Se trabaja con bits con alto conocimiento de la tarea y en esque- } \\
\text { ma fijo }\end{array}$ \\
\hline 7.- BITS+ UBICUIDAD & $\begin{array}{l}\text { Se trabaja con bits en esquema móvil y con bajo conocimiento de } \\
\text { la tarea }\end{array}$ \\
\hline 8.- C+U+B & $\begin{array}{l}\text { Se trabaja con bits, en esquema móvil y con alto requerimiento de } \\
\text { conocimiento para la tarea }\end{array}$ \\
\hline
\end{tabular}

El tercer elemento es la consolidación de los resultados de los informes PIT de los trabajadores de un grupo. Esta consolidación responde a esquemas diferentes dependiendo de la actividad de la organización (por proyectos, por clientes, por áreas funcionales...).

Con la metodología PIT, individual y grupal, se puede trabajar, de una forma práctica con grupos de trabajo y validar los fundamentos del modelo CUBOT. En la actualidad se está trabajando con varios grupos en empresas con distintos niveles de cercanía a modelos en red.

\section{Estudio de caso}

En este epígrafe se extraen las ideas principales del primer estudio de caso desarrollado como parte de esta línea de investigación. Para ello se incluye un breve perfil de la organización, los objetivos y metodología del caso, así como algunos resultados que se han obtenido.

Enred es una consultora española fundada en 1995 , dedicada al diseño, desarrollo, ejecución y evaluación de proyectos que contribuyen a la mejora de la sociedad en diferentes áreas:

- Igualdad de Oportunidades: estrategias y metodologías de mejora de la empleabilidad, fomento del emprendizaje femenino, inmigración, conciliación, acciones positivas en la empresa y transversalización de la perspectiva de género en la actividad de las organizaciones.

- Sociedad de la Información aplicada de un modo especializado a las necesidades del desarrollo (formación en red, e-servicios, trabajo en red y e-negocio, tanto concebidos como teleservicios internos dirigidos a agentes de intervención, como externos destinados a usuarios/as)

- Emprendizaje y mejora competitiva de la pyme: sistemas de apoyo para identificar y desarrollar los factores clave que permiten el refuerzo de su posición competitiva, desarrollo territorial, internacionalización, cooperación.

Su nombre, Enred, refleja además su filosofía de trabajo: cooperar, compartir, complementar, enriquecer proyectos y perspectivas a través del trabajo conjunto, configurando una red en la que se integran los colaboradores y organizaciones de apoyo.

La plantilla está estabilizada en torno a las 25 personas, la mayor parte consultores junior, senior o socios. El tipo de trabajo está centrado en el conocimiento, se apoya en sistemas de información (bits) y se puede realizar desde cualquier lugar y en cualquier momento (ubicuidad). 
El sistema de control de proyectos que se estableció en Enred, desde el inicio, se basa en:

- un informe individual de los consultores sobre su actividad mensual (ver anexo b),

- un análisis integrado por proyecto, por cliente, y por departamento de la dedicación de horas de la plantilla y de imputación de otros costes/gastos (ver anexo c).

Este sistema, tradicional en consultoras, es especialmente interesante en una consultora de tamaño mediano/pequeño porque aporta al consultor un análisis de su actividad en el marco de sus proyectos y su departamento.

Desde esta experiencia de 15 años, se establece como objetivo del estudio de caso analizar en profundidad el sistema de control de tiempos de Enred, su utilidad como modelo base de planificación integrada de tiempos, y su estandarización como metodología para otros casos en los que no se trabaje en consultoría.

Para cubrir este objetivo se ha seguido la siguiente metodología con elementos de análisis, cuantitativos y cualitativos:

I. Conocimiento del marco estratégico y organizativo de Enred en base a la información oficial de la organización, con el fin de tener definido un marco de referencia previo.

2. Realización del análisis de la estructura organizativa:

a) Organigrama y estructura de la empresa.

b) Cometido, objetivos y prioridades de las aéreas en que se divide.

c) Funciones asignadas a cada área y, en la medida de lo posible, a cada puesto de trabajo.

3. Análisis de los informes internos de seguimiento de actividad a nivel individual, de proyecto, de área y de empresa.

4. Evaluación del modelo y del nivel de satisfacción de los trabajadores con el mismo. Se facilitó un cuestionario para conocer el punto de partida del estudio. (Ver anexo a).

5. Evaluación del nivel de satisfacción de los socios con el sistema como herramienta de planificación y seguimiento de la actividad, con entrevistas en profundidad.
6. Evaluación de resultados e identificación de instrumentos de utilidad para la investigación de organización del trabajo en puestos del conocimiento. Ver anexos b y $c$.

Los resultados del cuestionario muestran que:

La flexibilidad en el puesto de trabajo es alta, encajando en los perfiles CUB del modelo CUBOT:

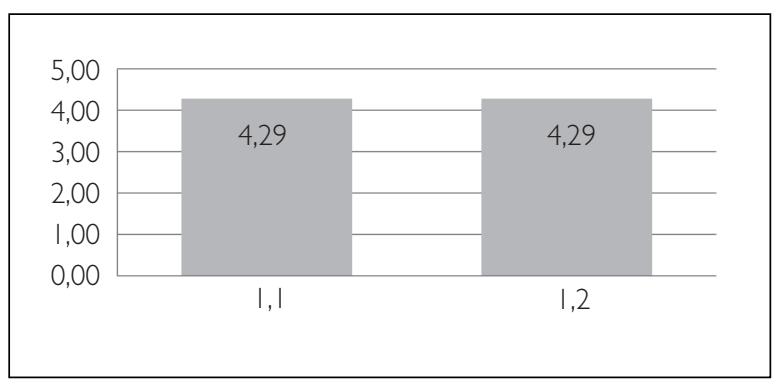

I. I: Mi puesto de trabajo me permite flexibilidad para trabajar fuera de la oficina (4.29)

1.2: Puedo organizar mi horario con libertad (4.29 sobre 5)

Los aspectos más valorados en relación a la caracterización de este tipo de de puestos son:

2.2: Considero que mi puesto de trabajo me permite aprender, desarrollarme y adaptarme a las circunstancias que lo rodean (4.14 sobre 5 )

2.5: Mi labor tiene importancia y repercusión en el conjunto de la organización (4.14)

2. I: Considero que la estructura y organización de mi puesto de trabajo me da autonomía para planificar, controlar y regular mi entorno. (4.00)

2.8: Llevo a cabo una planificación de mi trabajo diaria. (4.00)

Se detecta que el uso de la herramienta como parte del esquema de control de proyectos no implica necesariamente, que se aproveche como herramienta de mejora personal:

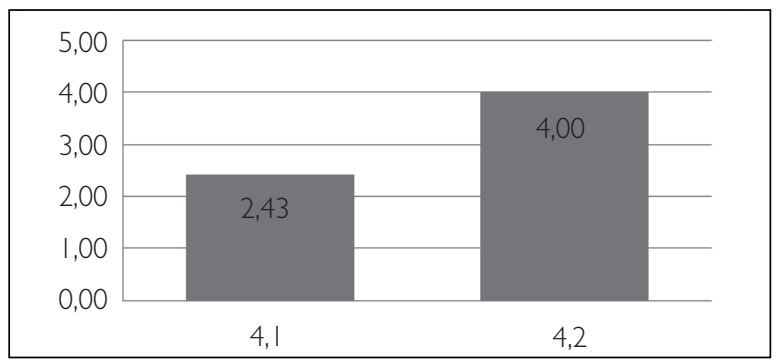


4. I: La información de seguimiento mensual es una herramienta que me ayuda a organizar mejor mí tiempo de trabajo (2.43)

4.2: Considero que la información de seguimiento mensual es una herramienta imprescindible para el control de los proyectos (4.0)

En cuanto a la valoración cualitativa de la validez del modelo cabe destacar las siguientes ideas extraídas de las entrevistas en profundidad a los socios:

- Avanzar en la cuantificación de horas, como unidad básica de trabajo del conocimiento, es de utilidad, sin embargo, los resultados obtenidos por hora de trabajo son distintos en cada persona, y eso es difícil de trasladar a números. La percepción directa del director del trabajador es básica para completar el análisis de productividad, y normalmente sus percepciones son bastante certeras.

- La aplicación sistemática de la herramienta de planificación de tiempos puede atenderse como una tarea burocrática, imprescindible para el control de proyectos integrado, pero sin aprovechar su potencial como instrumento para la planificación y control. Es necesario romper la tendencia a la burocratización para que sea una herramienta de utilidad individual.

- La información relacionada con la productividad agregada y el desarrollo de los proyectos debe llegar procesada al trabajador en un plazo de tiempo breve, ya que si no el retorno de la información no sirve para la toma de decisiones a todos los niveles.

Los resultados obtenidos de este estudio de caso se encuentran detallados en un informe que recoge los pasos de cada una de las fases de trabajo establecidas. Algunos de los aspectos técnicos y operativos son confidenciales. Cabe destacar que se han seleccionado dos instrumentos base para la aplicación de la metodología de planificación de tiempos integrada (ver anexos b y c) y que se han obtenido interesantes conclusiones a partir de la explotación del cuestionario de satisfacción y de las entrevistas a los socios.

El caso Enred, ha permitido constatar la validez del marco conceptual que se presenta en este artículo en un caso de empresa de conocimiento, a la vez que se ha podido evaluar, desde la perspectiva de trabajadores y directivos, la utilidad de las herramientas de planificación integrada de tiempos.

\section{Conclusiones}

Los primeros análisis teóricos, así como los estudios de caso que ya se han desarrollado y/o están en marcha, permiten anticipar que los puestos más cercanos a modelos en red (se trabaja con conocimiento, con alto nivel de complejidad y con flexibilidad espacial y temporal) requieren nuevos sistemas de planificación y seguimiento que permitan a los profesionales estructurar, dimensionar y evaluar las tareas en las que se desagregan sus funciones. La fragmentación que introduce la intensa conectividad de las organizaciones en red, hace esta tarea muy compleja. En ausencia de esquemas de ordenación consensuados de las funciones, de las tareas y de las asignaciones de tiempos a éstas, las respuestas del profesional pueden ser muy subjetivas y dificultar notablemente la función de dirección y coordinación en los equipos.

La mejora de la productividad que se espera de la incorporación de las TIC, sólo se confirmará si se avanza en la autentica transformación a modelos de trabajo en red. Las investigaciones sobre organizaciones en red tienen que ayudar a transformar la complejidad del entorno, en valor para las organizaciones y claridad en los puestos de trabajo.

El modelo de organización del trabajo en puestos del conocimiento que se ha presentado en este artículo, es un marco conceptual que permite empezar a caracterizar los puestos de trabajo de acuerdo a tres elementos: el modelo cubot que, a partir de tres variables significativas (desmaterizalización, nivel de conocimiento y ubicuidad) establece la cercanía a modelos de trabajo en red de un puesto de trabajo; la metodología de Planificación Integrada de Tiempos para que los trabajadores planifiquen, asignen y estimen los tiempos de sus diferentes actividades; y la integración grupal para que los directores de equipo hagan un análisis funcional agregado. Así mismo, la identificación de las dificultades que los trabajadores y trabajadoras pueden tener en ese tipo de puestos del conocimiento, permite establecer un modelo de planificación y mejora continua que refuerce sus competencias para su adecuado desempeño.

El estudio de caso de Enred muestra la validez del modelo CUBOT para la caracterización de los puestos de trabajo. También valida la utilización del método de planificación integrada de tiempos (PIT) como instrumento de ordenación en el puesto de trabajo individual y de evaluación de los grupos. Una de las conclusiones destacadas del caso, es que, si no 
se apoya a los trabajadores para que utilicen el PIT como una herramienta de planificación y mejora personal, se tenderá a burocratizar su uso y a ponerla sólo al servicio del control de los proyectos.

Con estos primeros resultados, se está revisando el modelo de organización del trabajo en puestos del conocimiento, y se están desarrollando nuevos estudios de caso, de mayor alcance, en Red Eléctrica de España y otras empresas no consultoras.

La motivación de estas empresas para implantar el modelo, coinciden con los supuestos de la investigación: sus trabajadores del conocimiento necesitan nuevos instrumentos para organizar mejor el trabajo, habitualmente fragmentado y con muchas interacciones, y así mejorar la productividad y disminuir el estrés.

\section{Bibliografía}

BARABÁSI, A.; Linked: How everything is connected to everything else and what it means. Plume Editors, 2002.

BROWN, S.J., DUGUID, P.; La vida social de la información, Buenos Aires, Prentice Hall, 2001.

BUENO, E.; Comisión de Trabajo de Gestión del Conocimiento Empresarial. CEDE, 2008.

CASADO, R. (Coord.), Claves de la alfabetización digital. Ariel, 2006.

CASTELLS, M.; La era de la información. Economía sociedad y cultura.Vol. 3. Fin del Milenio, Alianza Editorial, Madrid, 1998.

CONGDE; Informe de la CONGDE sobre el sector de las ONGD 2005. Especial temático: recursos humanos en las ONGD, Madrid, CONGDE, 2006.

HOLLINGSHEAD, A.; Communication Technologies, the Internet and Group Research. En M.A. Hogg, R. Scott, Blackwell Handbook of Social Psychology. Blackwell Publisher, 2001.

IGLESIAS-PRADAS, S., PASCUAL-MIGUEL, F., CHAPARRO-PELÁEZ, J., HERNÁNDEZ-GARCÍA. A., Influencia de las características del puesto de trabajo en la implantación de TIC móviles en la organización, Revista Dirección y Organización, N³ 38, 2009.

LUTHANS, F.; Comportamiento organizacional, McGrawHill, Nueva York, 2007.

MATAIX, C., MORENO,A.,ACEVEDO, M.; La adopción de estrategias y estructuras de red en ONGD. Revista Tercer Sector. Fundación Luis Vives, 2009.
MATAIX, C. MORENO, A., ACEVEDO, M.; Estructuras en red: diseño y modelos para el Tercer Sector, UNED, Fundación Luis Vives, Madrid, 2007.

MINTZBERG HENRY, Mintzberg on Management: Inside Our Strange World of Organizations, The Free Press, New York, NY, 1989. ISBN 0-02-92 I 37। - I, 1989

MINZBERG, H.; Diseño de organizaciones eficientes, Editorial El Ateneo, 1992.

MORENO, A., ACEVEDO, M.; Guía para la incorporación de las TIC en las intervenciones de educación en la cooperación al desarrollo , DGPOLDE - MAEC, 2008.

MORENO, A.; Organizaciones en red. Fundamentos psicosociales y de gestión del cambio. Sanz y Torres, 2009.

MORENO, A.; Adaptación a las nuevas tecnologías organizativas del trabajo en entornos de red. Telos. Cuadernos de comunicación e Innovación, Vol 8I, N ISSN: 02 | 3-084X, 2009.

MORENO, A.; TICs y la cooperación para el desarrollo y el manejo de la brecha digital desde la perspectiva de España. Las relaciones entre europa y latinoamerica en el contexto de la crisis economica mundial, SEGIB, 2009.

HOLLINGSHEAD, A.; Communication Technologies, the Internet and Group Research. En M.A. Hogg, R. Scott, Blackwell Handbook of Social Psychology, Blackwell Publisher, 200I.

MATAIX, C.; Dirección estratégica para las ONG, Madrid, UNED, 2001.

PEREZ, C.; Revoluciones tecnológicas y sistema financiero, Méjico, Siglo XXI Editores, 2005.

PÉREZ, J.; La Gobernanza de Internet y otras redes de conocimiento. Fundación Telefónica, ETSIT-UPM ISBN: 97884-08-08|8|-4, 2008.

SANTOS, J.I., GALÁN, J.M, IZQUIERDO, L., OLMO, R., Aplicaciones de las TIC en el nuevo modelo de enseñanza del EEES, Revista Dirección y Organización. No 39, 2009.

SENNETT, R.; La corrosión del carácter. Las consecuencias personales del trabajo en el nuevo capitalismo, Editorial Anagrama, 1998.

SPEARS, R., LEA, M.; Panacea or Panopticon? The Hidden Power in Computer-Mediated Communication, Communication Research, 21, 4, 427-459, 1994.

TOPA, G., MORENO, A., URIARTE, L.M.; La responsabilidad social corporativa en las empresas. La empresa mestiza, Aula Solidaridad, www.entredosorillas.org, 2006.

WELLMAN, B.; Living Networked in a Wired World:The Persistence and Transformation of Community, Report to the Law Commission of Canada, Wellman Associates, Toronto, Canada, 2001. 
WIESENFIELD, B., REGHURAM, S., GARUD, R.;, «Organizational identification among virtual workers: the role of need for affiliation and perceived work-based social support», Journal of Management, 27, 213-229, 2001.
ZORNOZA,A., ORENGO,V., GOSALVEZ, I., GONZÁLEZ, P.; Teamwork in different communication contexts: a longitudinal study, Psychology in Spain, 2002, Vol. 6. No I, 4I-55. Colegio Oficial de Psicólogos, 2002.

\title{
Anexo A: Cuestionario de caracterización y satisfacción de trabajadores red
}

\section{CUESTIONARIO-ORGANIZACIÓN DEL TRABAJO EN PUESTOS DEL CONOCIMIENTO}

\author{
BLOQUE O - INFORMACIÓN GENERAL
}

Edad:

Sexo:

Compañía:

Puesto que ocupa:

Número de personas que tiene a su cargo:

\section{BLOQUE I - CARACTERIZACIÓN DEL PUESTO DETRABAJO}

Para las cuestiones 1.1 y 1.2 valore su grado de conformidad de la siguiente manera: I - Nada, 2 - Poco, 3 - Medio, 4 - Bastante, 5 - Mucho

\begin{tabular}{|l|l|l|l|l|c|}
\hline \multicolumn{1}{|c|}{ Preguntas } & \multicolumn{4}{|c|}{ Importancia } \\
\hline \multicolumn{1}{|c|}{ Escala } & I & $\mathbf{2}$ & $\mathbf{3}$ & $\mathbf{4}$ & $\mathbf{5}$ \\
\hline $\begin{array}{l}\text { I.I Mi puesto de trabajo me permite flexibilidad para trabajar fuera de la } \\
\text { oficina. }\end{array}$ & & & & & \\
\hline I.2. Puedo organizar mi horario con libertad. & & & & & \\
\hline
\end{tabular}

Para las cuestiones 1.3, I.4 y 1.5 indique el porcentaje en el que se cumplen cada una de las afirmaciones, siendo este una estimación aproximada.

\begin{tabular}{|l|l|l|l|l|l|l|l|l|l|l|l|l|}
\hline \multicolumn{1}{|c|}{ Preguntas } & \multicolumn{7}{c|}{ Porcentaje (\%) } \\
\hline \multicolumn{1}{|c|}{ Escala } & $\mathbf{0}$ & $\mathbf{1 0}$ & $\mathbf{2 0}$ & $\mathbf{3 0}$ & $\mathbf{4 0}$ & $\mathbf{5 0}$ & $\mathbf{6 0}$ & $\mathbf{7 0}$ & $\mathbf{8 0}$ & $\mathbf{9 0}$ & $\mathbf{1 0 0}$ \\
\hline $\begin{array}{l}\text { I.3 iQué proporción de su trabajo está vinculado a } \\
\text { información y conocimiento que se cuentra en formato } \\
\text { digital? }\end{array}$ & & & & & & & & & & & \\
\hline $\begin{array}{l}\text { I.4 ¿Qué porcentaje de tiempo dedica a interacción con } \\
\text { otras personas de forma digital? }\end{array}$ & & & & & & & & & & & \\
\hline $\begin{array}{l}\text { I.5 ¿Qué porcentaje cree que deba ser obligatoriamente } \\
\text { cara a cara? }\end{array}$ & & & & & & & & & & & \\
\hline
\end{tabular}




\section{BLOQUE 2 - ORGANIZACIÓN DEL PUESTO DE TRABAJO}

Para cada una de las consideraciones siguientes valore su grado de conformidad de la siguiente manera: I - Nada, 2 - Poco, 3 - Medio, 4 - Bastante, 5 - Mucho

\begin{tabular}{|c|c|c|c|c|c|}
\hline Preguntas & \multirow[b]{2}{*}{$\mathbf{I}$} & \multirow[b]{2}{*}{2} & \multirow[b]{2}{*}{3} & \multirow[b]{2}{*}{4} & \multirow[b]{2}{*}{5} \\
\hline Escala & & & & & \\
\hline $\begin{array}{l}\text { 2.I Considero que la estructura y organización de mi puesto de trabajo me da } \\
\text { autonomía para planificar, controlar y regular mi entorno. }\end{array}$ & & & & & \\
\hline $\begin{array}{l}\text { 2.2 Considero que mi puesto de trabajo me permite aprender, desarrollarme y } \\
\text { adaptarme a las circunstancias que lo rodean. }\end{array}$ & & & & & \\
\hline $\begin{array}{l}\text { 2.3 Considero que mi puesto de trabajo ofrece variedad de experiencias o actividades, } \\
\text { por lo que no puede considerarse repetitivo. }\end{array}$ & & & & & \\
\hline $\begin{array}{l}\text { 2.4 Me siento partícipe a la hora de diseñar o planificar el contenido del puesto, o los } \\
\text { cambios que se hacen en el mismo. }\end{array}$ & & & & & \\
\hline $\begin{array}{l}2.5 \text { Considero que mi labor tiene la importancia y repercusión en el conjunto de la } \\
\text { organización. }\end{array}$ & & & & & \\
\hline 2.6 Llevo a cabo una planificación de mi trabajo anual. & & & & & \\
\hline 2.7 Llevo a cabo una planificación de mi trabajo mensual. & & & & & \\
\hline 2.8 Llevo a cabo una planificación de mi trabajoj diaria. & & & & & \\
\hline 2.9 Consigo que la realidad se ajuste a lo planificado. & & & & & \\
\hline 2.10 Las reuniones no planificadas son un elemento distorsionador de la planificación. & & & & & \\
\hline 2.II El teléfono electrónico tiene un impacto negativo en la planificación. & & & & & \\
\hline 2.12 El correo electrónico tiene un impacto negativo en la planificación & & & & & \\
\hline 2.13 La cantidad de trabajo desborda el tiempo de mi jornada laboral. & & & & & \\
\hline 2.14 Considero que trabajar con ciertos niveles de estrés es inevitable. & & & & & \\
\hline
\end{tabular}

\section{BLOQUE 3 - UTILIDAD DE LA PLANIFICACIÓN}

Para cada una de las consideraciones siguientes valore su grado de conformidad de la siguiente manera: I - Nada, 2 - Poco, 3 - Medio, 4 - Bastante, 5 - Mucho

\begin{tabular}{|l|l|l|l|l|l|}
\hline \multicolumn{1}{|c|}{ Preguntas } & \multicolumn{4}{c|}{ Importancia } \\
\hline \multicolumn{1}{|c|}{ Escala } & I & $\mathbf{2}$ & $\mathbf{3}$ & $\mathbf{4}$ & $\mathbf{5}$ \\
\hline 3.I Soy capaz de estimar el tiempo que dedico a cada una de mis acciones. & & & & & \\
\hline $\begin{array}{l}\text { 3.2 Sería de utilidad tener una herramienta corporativa para saber el tiempo } \\
\text { que dedico a cada tarea. }\end{array}$ & & & & \\
\hline $\begin{array}{l}\text { 3.3 Personalmente creo interesante dedicar parte de mi tiempo a la mejora } \\
\text { de los sistemas de planificación individual y del grupo. }\end{array}$ & & & & & \\
\hline
\end{tabular}




\section{BLOQUE 4 - UTILIDAD DE LA PLANIFICACION MENSUAL}

Para las cuestiones 4.1 y 42 valore su grado de conformidad de la siguiente manera: I - Nada, 2 - Poco, 3 - Medio, 4 - Bastante, 5 - Mucho

\begin{tabular}{|l|l|l|l|l|c|}
\hline \multicolumn{1}{|c|}{ Preguntas } & \multicolumn{4}{|c|}{ Importancia } \\
\hline \multicolumn{1}{|c|}{ Escala } & I & $\mathbf{2}$ & $\mathbf{3}$ & $\mathbf{4}$ & $\mathbf{5}$ \\
\hline $\begin{array}{l}\text { 4.I La información de seguimiento mensual es una herramienta que me ayuda } \\
\text { a organizar mejor mi tiempo de trabajo. }\end{array}$ & & & & \\
\hline $\begin{array}{l}\text { 4.2 Considero que la información de seguimiento mensual es una herramienta } \\
\text { imprescindible para el control de los proyectos. }\end{array}$ & & & & \\
\hline
\end{tabular}

Para las cuestiones 4.3,4.4 indique el porcentaje en el que se cumplen cada una de las afirmaciones, siendo este una estimación aproximada.

\begin{tabular}{|l|l|l|l|l|l|l|l|l|l|l|l|l|}
\hline \multicolumn{2}{|c|}{ Preguntas } & \multicolumn{7}{|c|}{ Porcentaje (\%) } \\
\hline \multicolumn{1}{|c|}{ Escala } & $\mathbf{0}$ & $\mathbf{1 0}$ & $\mathbf{2 0}$ & $\mathbf{3 0}$ & $\mathbf{4 0}$ & $\mathbf{5 0}$ & $\mathbf{6 0}$ & $\mathbf{7 0}$ & $\mathbf{8 0}$ & $\mathbf{9 0}$ & $\mathbf{1 0 0}$ \\
\hline $\begin{array}{l}4.3 \text { iEn qué porcentaje aprovechas la información de los } \\
\text { informes de mes (Highlights report) para planificar tu } \\
\text { trabajo? }\end{array}$ & & & & & & & & & & & \\
\hline $\begin{array}{l}4.4 \text { ¿En qué porcentaje aprovechas la información de los } \\
\text { informes de mes (Highlights report) para evaluar tu } \\
\text { trabajo? }\end{array}$ & & & & & & & & & & & \\
\hline
\end{tabular}

\section{Anexo B: Plantilla para la planificación integrada de tiempos}

\section{INFORME MENSUAL}

Persona:

Mes:

\begin{tabular}{|c|c|c|c|c|c|c|c|c|c|}
\hline \multirow[t]{2}{*}{ Proyecto/Tarea } & \multicolumn{5}{|c|}{ Horas } & \multirow[t]{2}{*}{ Objetivos/Mes } & \multirow[t]{2}{*}{$\begin{array}{l}\text { Resultados } \\
\text { alcanzados }\end{array}$} & \multirow{2}{*}{$\begin{array}{l}\text { Objetivos Mes } \\
\text { siguiente }\end{array}$} & \multirow[t]{2}{*}{$\begin{array}{l}\text { Comentarios } \\
\text { Desviaciones }\end{array}$} \\
\hline & estimado & IS & $2 S$ & 35 & 45 & & & & \\
\hline \multicolumn{10}{|l|}{ Proyecto I } \\
\hline \multicolumn{10}{|l|}{ Actividad I } \\
\hline \multicolumn{10}{|l|}{ Actividad 2.. } \\
\hline \multicolumn{10}{|l|}{ Proyecto 2} \\
\hline \multicolumn{10}{|l|}{ Actividad I } \\
\hline \multicolumn{10}{|l|}{ Actividad 2} \\
\hline \multicolumn{10}{|l|}{ Proyecto 3} \\
\hline \multicolumn{10}{|l|}{ Actividad I } \\
\hline \multicolumn{10}{|l|}{ Actividad 2} \\
\hline TOTAL & & & & & & & & & \\
\hline
\end{tabular}




\section{Anexo C: Informe de proyecto a partir de informes mensuales individuales}

CLIENTE:

TITULO PROYECTO:

CÓDIGO PROYECTO:
IMPORTE CONTRATO

Evaluación:

Pendiente de Producir

Pendiente de Facturar

Pendiente de Cobro

\begin{tabular}{|l|l|l|l|l|l|l|l|l|l|l|l|l|}
\hline & Acumulado & Enero & Febrero & Marzo & Abril & ... & Diciembre & Totales & \multicolumn{2}{|l|}{} \\
\hline Producción Real & & & & & & & & & \multicolumn{2}{|l}{ Facturación Consumida } \\
\hline Facturación & & & & & & & & & & & Coste Medio/Hora & \\
\hline Producción Objetivo & & & & & & & & & & & Facturación Medio/Hora & \\
\hline Total Gastos & & & & & & & & & & & \\
\hline
\end{tabular}

\begin{tabular}{|l|l|l|l|l|l|l|l|l|}
\hline \multicolumn{1}{|c|}{ PERSONAL } & & & & & & & & Total Horas \\
\hline \#Nombre_1 & & & & & & & & \\
\hline \#Nombre_2 & & & & & & & & \\
\hline \#Nombre_3 & & & & & & & & \\
\hline.-- & & & & & & & & \\
\hline \#Nombre_n & & & & & & & & \\
\hline Tot.Coste Personal & & & & & & & & \\
\hline Tot. Horas & & & & & & & & \\
\hline
\end{tabular}

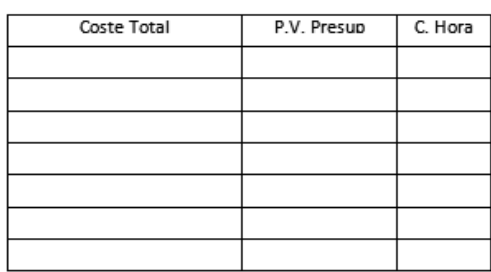

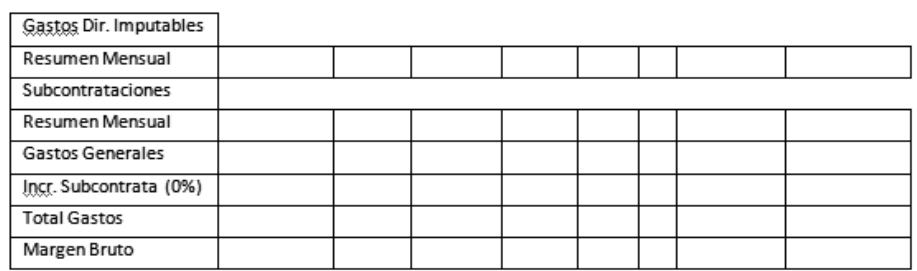

T. Gastos Directos
\begin{tabular}{|c|l|l|}
\hline \multicolumn{2}{|l|}{} & \\
\hline T. Subcontrataciones & & \\
\hline T.Gastos Generales & & \\
\hline T. Incremento Subcont & & \\
\hline Total Gastos & & \\
\hline Total Margen bruto & & \\
\hline & $\begin{array}{l}\text { Margen Bruto/ } \\
\text { Producción real }\end{array}$ & \\
\hline
\end{tabular}

